\title{
Declines in Births by Month: United States, 2020
}

\author{
Brady E. Hamilton, Ph.D., Michelle J.K. Osterman, M.H.S., and Joyce A. Martin, M.P.H., \\ Division of Vital Statistics, National Center for Health Statistics
}

\section{Ahstract}

Objectives - This report presents provisional 2020 and final 2019 and 2018 data on changes in the number of U.S. births by race and Hispanic origin of mother and by month of birth and state.

Methods - Data are based on all birth certificates registered to U.S. residents in the 50 states and D.C.; data for 2020 are provisional and data for 2018 and 2019 are final. Changes in the number of births by month from 2019 to 2020 by race and Hispanic-origin group are compared with changes occurring from 2018 to 2019. Changes for the first and second 6 months of the year by race and Hispanic origin and by state are also compared for the periods 2019 to 2020 and 2018 to 2019 .

Results-From 2019 to 2020, the number of births for the United States declined for each month, with the largest declines occurring in December (8\%), August (7\%), and October and November (6\%) (Figure 1). Larger declines in births were seen in the second half of 2020 (down 6\%) compared with the first half (down 2\%) of 2020. The number of births declined in both the first and second 6 months of 2020 compared with 2019 for nearly all race and Hispanic-origin groups, with larger declines in the second half of 2020 compared with the first half of the year. Births declined in 20 states in the first half of 2020, and in all states in the second half of 2020 (declines in 7 states were not significant). Changes in births by race and Hispanic origin and by state were less pronounced from 2018 to 2019; the number of births declined for 9 months by $1 \%-3 \%$.
Keywords: birth certificate $\bullet$ maternal race and Hispanic origin $\bullet$ state $\bullet$ National Vital Statistics System

\section{Introduction}

On March 13, 2020, the U.S. government declared a national emergency in response to the emergence of the COVID-19 pandemic (1). The pandemic, which is ongoing as of the publication of this report, has constituted a public health event not seen in the country since the 1918 influenza pandemic (2). A recent report has shown a decline of 1.0 year in the overall life expectancy at birth in the first 6 months of 2020 (3). The impact of the pandemic on trends in fertility for the United States has been uncertain, with predictions ranging from an upswing to a severe decline (4-6).
Births generally had been on the decline in the United States from 2007 to 2019, with declines averaging about $2 \%$ annually (7-10). A recent report showed a $4 \%$ drop in the total number of U.S. births from 2019 to 2020, the largest annual decline in the number of births since 1973 (7-11). The number of births declined for all race and Hispanic-origin groups from 2019 to 2020, with declines ranging from $3 \%$ (Hispanic women) to 8\% (non-Hispanic Asian women) (7). The U.S. general fertility rate (GFR) also declined 4\% from 2019 to 2020 , the largest percent decline in this rate in nearly five decades; rates declined for each race and Hispanic-origin group (7).

The impact of the pandemic on the United States in 2020 varied by month, as reported infection cases rose rapidly through the year, from 26 cases in early March to over 20 million cases at

Figure 1. Percent change in number of births, by month: United States, 2019 final and 2020 provisional

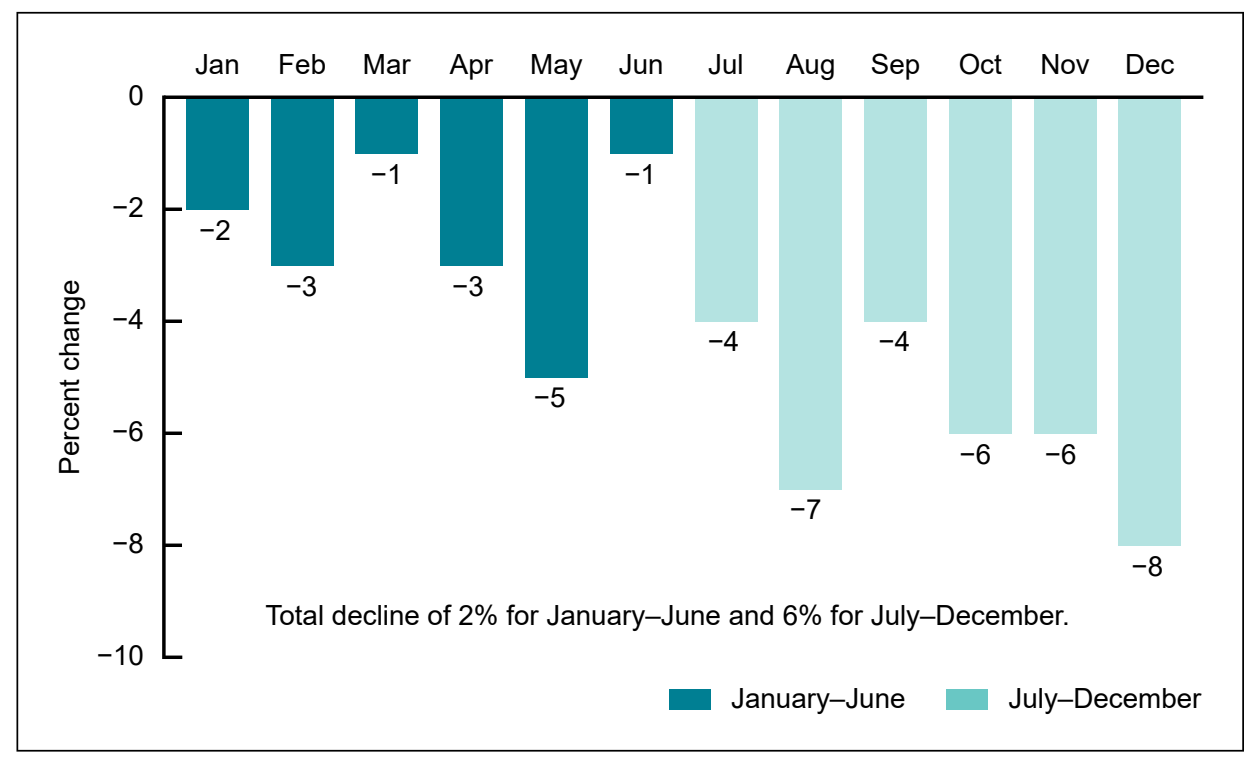

NOTES: Number of births for February 2020 was adjusted for leap day, February 29. Significant declines from 2019 to 2020 for all months $(p<0.05)$.

SOURCE: National Center for Health Statistics, National Vital Statistics System, Natality. 
the end of December. The impact also varied widely by state as the infection spread across the United States, from 7 states reporting confirmed cases in early March to all 50 states, D.C., and the territories (except American Samoa) reporting confirmed cases by the end of March (12). This report examines changes occurring in the number of U.S. births by race and Hispanic origin by single month and for the first and second 6 months (January-June and July-December) of 2020, and in the number of births by state of residence for the first and second 6 months of 2020. Changes are compared with those occurring from 2018 to 2019, with the focus on the changes from 2019 to 2020 , encompassing the period when the pandemic arose.

\section{Methods}

Data for 2020 are based on $99.87 \%$ of all birth records received and processed by the National Center for Health Statistics as of February 11, $2021(7,8,13)$. The number of provisional 2020 births are compared with final 2019 counts. Changes in final birth counts from 2018 to 2019 are also described.

Hispanic origin and race are reported separately on the birth certificate. Data shown by Hispanic origin include all persons of Hispanic origin of any race. Data for non-Hispanic persons are shown separately for each single-race group. Data by race are based on the revised standards issued by the Office of Management and Budget in 1997 (14). The race and Hispanic-origin groups shown are non-Hispanic, single-race white; non-Hispanic, single-race black; non-Hispanic, single-race American Indian or Alaska Native (AIAN); non-Hispanic, single-race Asian; non-Hispanic, single-race Native Hawaiian or Other Pacific Islander (NHOPI); and Hispanic. For brevity, text references to race omit the term "single-race" (10).

The number of births for February 2020 was adjusted to account for the additional day in February 2020, compared with February 2019 (that is, for the leap day, February 29). Both observed and adjusted numbers are shown for February 2020 in Table 1; text comparisons are based on the adjusted February 2020 number of births. The number of births for the total year and for the first half of 2020 were not adjusted for leap day due to the relatively small impact of the additional births on February 29.

Births by state by month were not analyzed because of the small number of births by month for some states.

All changes in the number of births and differences in the changes mentioned in this report are statistically significant at the 0.05 level based on a chi-squared test, unless noted otherwise.

\section{Results}

\section{Changes in the number of births by month}

- From 2019 to 2020, the number of births declined for each month, with the largest declines in December (8\%), August (7\%), and October and November $(6 \%)$ (Table 1 and Figure 1). In comparison, from 2018 to 2019 , the number of births declined for 9 months of the year, with declines ranging from $1 \%$ to $3 \%$.
- The decline in the number of births between 2019 and 2020 was larger in the second half of the year $(6 \%)$ than in the first half of the year $(2 \%)$. Between 2018 and 2019, the rate declined $2 \%$ in the first half of the year and $1 \%$ in the second half. (Table 1) $(10,15)$.

\section{Changes in the number of births by maternal race and Hispanic origin}

- From 2019 to 2020, declines in the number of births by month for non-Hispanic white women ranged from $1 \%$ to $7 \%$, for non-Hispanic black women from $2 \%$ to $8 \%$, and for Hispanic women from $2 \%$ to $9 \%$ (Table 1). For non-Hispanic AIAN and non-Hispanic Asian women, declines in births by month ranged from $6 \%$ to $13 \%$ and from $3 \%$ to $19 \%$, respectively. Among non-Hispanic NHOPI women, changes in births by month from 2019 to 2020 were not significant. From 2018 to 2019 , monthly changes among the maternal race and Hispanic-origin groups ranged from declines of $2 \%$ to $9 \%$ to increases of $1 \%$ to $12 \%$.

- The decline in births between 2019 and 2020 was larger in the second

Figure 2. Percent change in number of births, by race and Hispanic origin: United States, January-June, 2019 final and 2020 provisional; and July-December, 2019 final and 2020 provisional

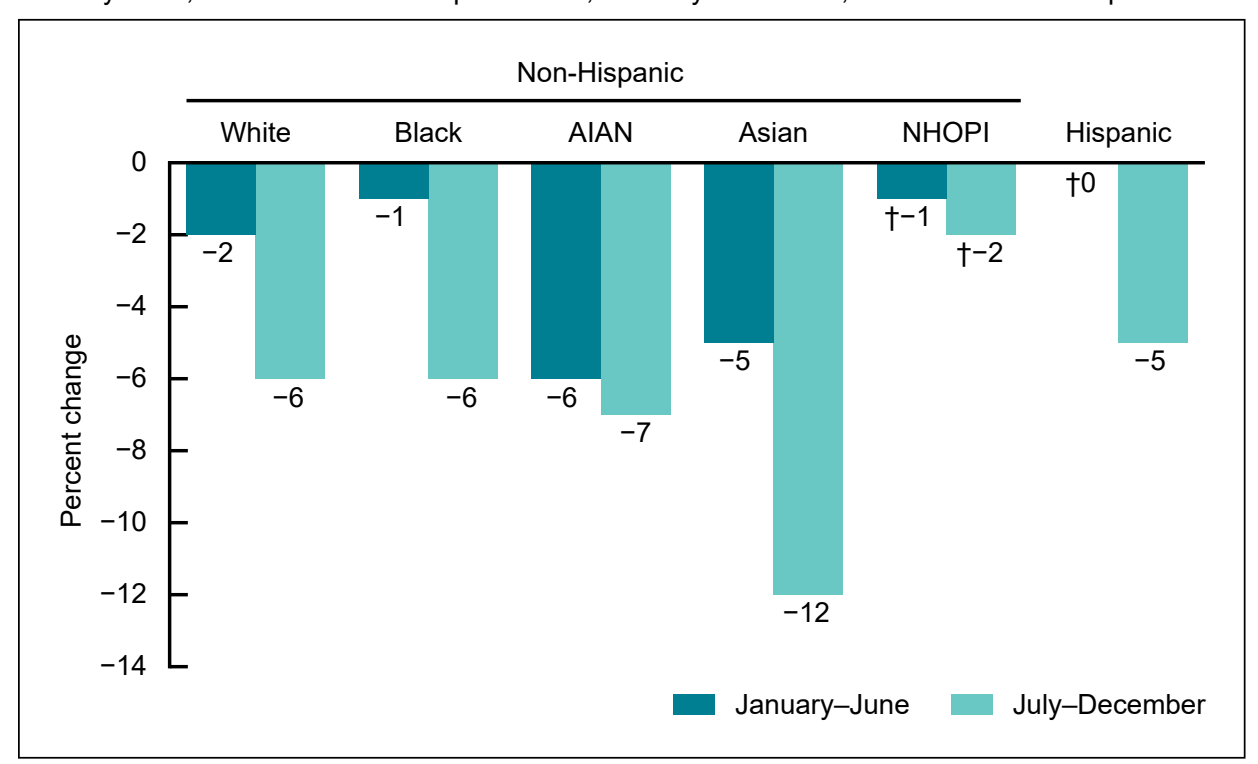

† No significant change $(p<0.05)$

NOTES: AIAN is American Indian or Alaska Native. NHOPI is Native Hawaiian or Other Pacific Islander. SOURCE: National Center for Health Statistics, National Vital Statistics System, Natality. 
half of the year than in the first half for non-Hispanic white $6 \%$ and $2 \%$, respectively), non-Hispanic black (6\% and 1\%), non-Hispanic Asian (12\% and 5\%), and Hispanic (5\% and $0 \%$ ) women (Table 1 and Figure 2). The difference in the declines in the number of births for non-Hispanic AIAN (7\% and 6\%) and non-Hispanic NHOPI ( $2 \%$ and $1 \%$ ) women were not significant.

\section{Decline in number of births by state}

- For the first half of 2020, 20 states reported declines in the number of births compared with the same period in 2019, with 30 states and D.C. reporting no significant change. Similarly, between the first half of 2018 and the first half of 2019, the number of births declined for 27 states, did not change significantly for 22 states or D.C., and increased for 1 state.

- For the second half of 2020, births declined for all states and D.C. compared with the same period in 2019 (declines in seven states were not significant) (Table 2 and Figure 3). In contrast, during the second 6 months of 2018 and 2019, births declined for 9 states, increased for 5 states, and did not change significantly for 36 states and D.C.

- The largest declines by state for the second half of 2020 were seen in New Mexico (11\%), New York (9\%), and California, Hawaii, and West Virginia (8\% each); 38 states and D.C. had declines of $3 \%$ to $7 \%$ (Table 2 ).

\section{Summary}

From 2019 to 2020, the number of births declined for each month by $1 \%$ to $8 \%$, with larger declines observed for the second half of $2020(6 \%)$ compared with the first half of the year (2\%). Births declined for all race and Hispanic-origin groups in both the first and second half of 2020, with larger declines in the second half of the year compared with the first half for all groups (the difference in the declines in births to

Figure 3. Percent change in number of births, by state of residence: United States, January-June, 2019 final and 2020 provisional; and July-December, 2019 final and 2020 provisional

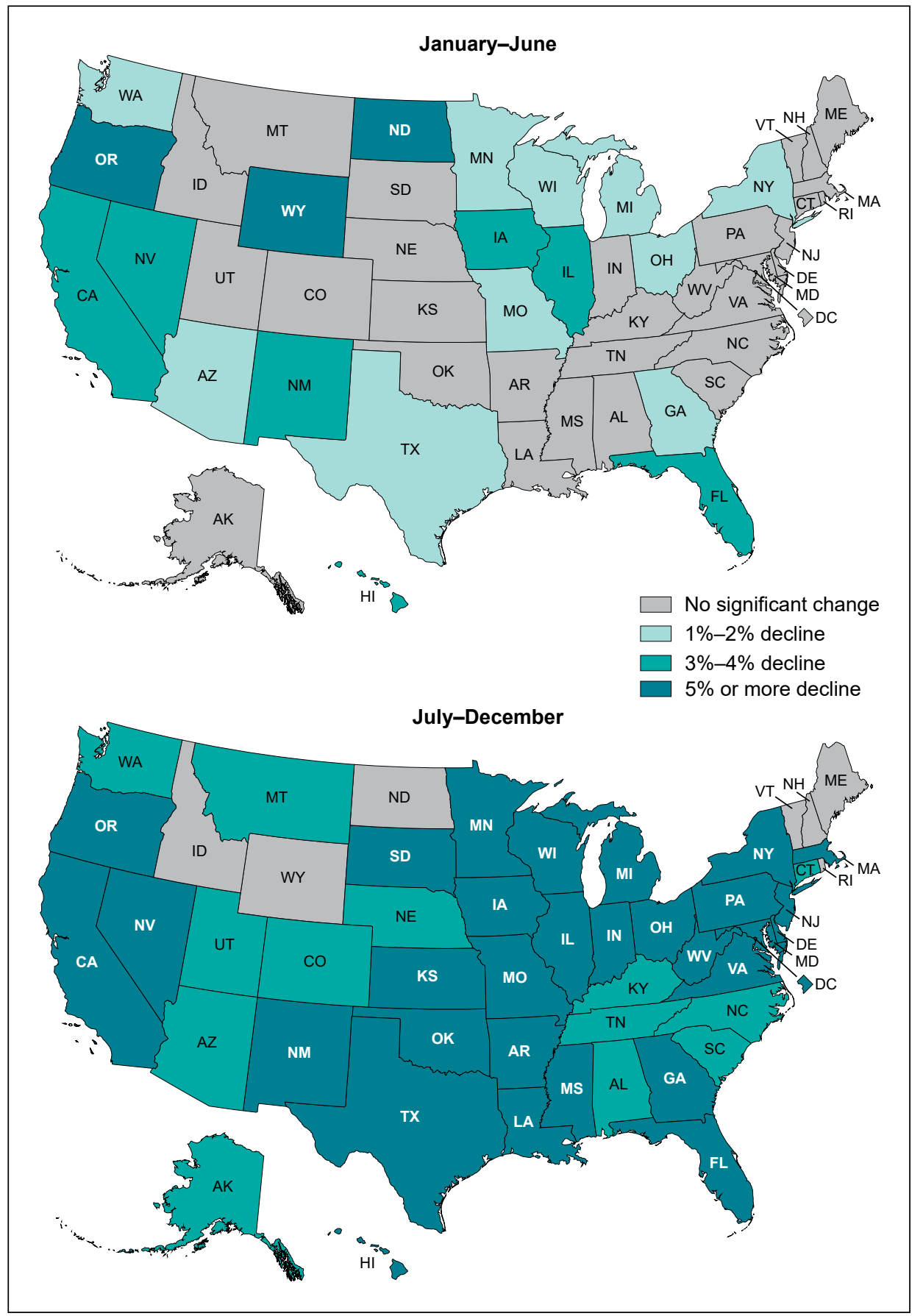

SOURCE: National Center for Health Statistics, National Vital Statistics System, Natality.

non-Hispanic AIAN and NHOPI women was not significant). The number of births declined for all states in the second half of 2020 (declines for 7 states were not significant), compared with declines for only 20 states in the first half of 2020 and 9 states in the second half of 2019.

Evaluation of trends in births by month will continue to determine whether these declines continued into 2021 or were unique to 2020 during the time of the initial COVID-19 pandemic. 


\section{References}

1. Executive Office of the President. Declaring a national emergency concerning the novel coronavirus disease (COVID-19) outbreak. Proclamation 9994. Fed Regist 85(53):15337-8. 2020. Available from: https://www.govinfo.gov/ content/pkg/FR-2020-03-18/ pdf/2020-05794.pdf.

2. Cheema S, Ameduri M, Abraham A, Doraiswamy S, Mamtani R. The COVID-19 pandemic: The public health reality. Epidemiol Infect 148:e223. 2020. Available from: https://www.ncbi.nlm.nih.gov/pmc/ articles/PMC7533474.

3. Arias E, Tejada-Vera B, Ahmad F. Provisional life expectancy estimates for January through June, 2020. Vital Statistics Rapid Release; no 10. Hyattsville, MD: National Center for Health Statistics. February 2021. Available from: https://www.cdc. gov/nchs/data/vsrr/VSRR10-508.pdf.

4. Kearney MS, Levine P. Half a million fewer children? The coming COVID baby bust. Brookings Institution. 2020. Available from: https://www.brookings.edu/research/ half-a-million-fewer-children-thecoming-covid-baby-bust/.

5. Kearney MS, Levine P. The coming COVID-19 baby bust: Update. Brookings Institution. 2020. Available from: https:// www.brookings.edu/blog/upfront/2020/12/17/the-coming-covid19-baby-bust-update/.

6. Lindberg LD. The coming COVID baby bust. Guttmacher Institute. 2021. Available from: https://www. guttmacher.org/article/2021/01/ coming-covid-baby-bust.

7. Hamilton BE, Martin JA, Osterman MJK. Births: Provisional data for 2020. Vital Statistics Rapid Release; no 12. Hyattsville, MD: National Center for Health Statistics. May 2021. Available from: https://www. cdc.gov/nchs/data/vsrr/vsrr012-508. pdf.
8. Driscoll AK, Osterman MJK, Hamilton BE, Martin JA. Quarterly provisional estimates for selected birth indicators, 2018-Quarter 4, 2020. National Center for Health Statistics. National Vital Statistics System, Vital Statistics Rapid Release Program. 2021. Available from: https://www.cdc.gov/nchs/ nvss/vsrr/natality.htm.

9. National Center for Health Statistics, National Vital Statistics System. Provisional estimates for selected maternal and infant outcomes by month, 2018-2020. Hyattsville, MD. Available from: https://www.cdc. gov/nchs/covid19/technical-notesoutcomes.htm.

10. Martin JA, Hamilton BE, Osterman MJK, Driscoll AK. Births: Final data for 2019. National Vital Statistics Reports; vol 70 no 2. Hyattsville, MD: National Center for Health Statistics. 2021. Available from: https://www.cdc.gov/nchs/data/nvsr/ nvsr70/nvsr70-02-508.pdf.

11. Martin JA, Hamilton BE, Osterman MJK, Driscoll AK, Mathews TJ. Births: Final data for 2015. National Vital Statistics Reports; vol 66 no 1. Hyattsville, MD: National Center for Health Statistics. 2017. Available from: https://www.cdc.gov/nchs/ data/nvsr/nvsr66/nvsr66_01.pdf.

12. Centers for Disease Control and Prevention. COVID data tracker. Available from: https://covid.cdc.gov/ covid-data-tracker/\#datatracker-home.

13. National Center for Health Statistics. Quarterly Provisional EstimatesTechnical Notes-Natality, Quarter 4, 2020. Available from: https://www. cdc.gov/nchs/nvss/vsrr/natalitytechnical-notes.htm.

14. Office of Management and Budget. Revisions to the standards for the classification of federal data on race and ethnicity. Fed Regist 62(210):58782-90. 1997. Available from: https://www.govinfo.gov/ content/pkg/FR-1997-10-30/pdf/9728653.pdf.
15. Martin JA, Hamilton BE, Osterman MJK, Driscoll AK. Births: Final data for 2018. National Vital Statistics Reports; vol 68 no 13. Hyattsville, MD: National Center for Health Statistics. 2019. Available from: https://www.cdc.gov/nchs/data/nvsr/ nvsr68/nvsr68_13-508.pdf.

\section{List of Detailed Tables}

\section{Report tables}

1. Births, by race and Hispanic origin of the mother and month of birth: United States, final 2018 and 2019 and provisional 2020, and percent change in births from 2018-2019 and 2019-2020. . . . . . . . . 5

2. Births: United States and each state, January-June and July-December, final 2018 and 2019 and provisional 2020 , and percent change in births from January-June and July-December, 2018-2019 and 2019-2020. ............ 7 
Vital Statistics Surveillance Report

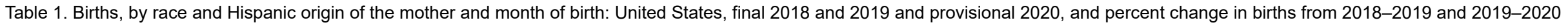

\begin{tabular}{|c|c|c|c|c|c|c|c|c|c|c|c|c|c|c|c|c|}
\hline $\begin{array}{c}\text { Race and } \\
\text { Hispanic origin } \\
\text { of mother and } \\
\text { year }\end{array}$ & Total & $\begin{array}{l}\text { January- } \\
\text { June }\end{array}$ & $\begin{array}{c}\text { July- } \\
\text { December }\end{array}$ & January & $\begin{array}{l}\text { February } \\
\text { (observed) }\end{array}$ & $\begin{array}{c}\text { February } \\
\text { (adjusted) }{ }^{1}\end{array}$ & March & April & May & June & July & August & September & October & November & December \\
\hline $\begin{array}{c}\text { All races and } \\
\text { origins }^{2}\end{array}$ & \multicolumn{16}{|c|}{ Number } \\
\hline $2020^{3}$ & $3,605,201$ & $1,782,237$ & $1,822,964$ & 304,683 & 282,601 & 272,856 & 301,457 & 290,252 & 301,313 & 301,931 & 321,253 & 319,313 & 311,224 & 304,460 & 281,576 & 285,138 \\
\hline 2019 & $3,747,540$ & $1,814,497$ & $1,933,043$ & 310,872 & 279,963 & 279,963 & 304,237 & 298,947 & 316,386 & 304,092 & 333,646 & 341,685 & 325,781 & 325,043 & 298,086 & 308,802 \\
\hline 2018 & $3,791,712$ & $1,848,934$ & $1,942,778$ & 314,808 & 284,250 & 284,250 & 316,044 & 298,394 & 320,622 & 314,816 & 329,009 & 344,750 & 322,772 & 326,778 & 308,703 & 310,766 \\
\hline \multicolumn{17}{|l|}{$\begin{array}{l}\text { Non-Hispanic, } \\
\text { single race }\end{array}$} \\
\hline \multicolumn{17}{|l|}{ White: } \\
\hline $2020^{3}$ & $1,839,565$ & 916,442 & 923,123 & 152,502 & 143,676 & 138,722 & 155,886 & 150,811 & 156,788 & 156,779 & 164,990 & 162,403 & 157,874 & 154,074 & 139,928 & 143,854 \\
\hline 2019 & $1,915,912$ & 937,741 & 978,171 & 156,819 & 142,992 & 142,992 & 157,502 & 156,516 & 165,587 & 158,325 & 172,130 & 174,284 & 165,366 & 165,035 & 148,646 & 152,710 \\
\hline 2018 & $1,956,413$ & 961,247 & 995,166 & 159,323 & 145,906 & 145,906 & 164,884 & 157,306 & 168,974 & 164,854 & 170,622 & 178,368 & 165,502 & 168,336 & 156,411 & 155,927 \\
\hline \multicolumn{17}{|l|}{ Black: } \\
\hline $2020^{3}$ & 528,448 & 259,621 & 268,827 & 46,344 & 42,029 & 40,580 & 43,560 & 41,364 & 42,980 & 43,344 & 46,367 & 46,744 & 45,188 & 44,134 & 42,446 & 43,948 \\
\hline 2019 & 548,075 & 262,114 & 285,961 & 47,486 & 41,497 & 41,497 & 43,583 & 42,151 & 44,584 & 42,813 & 48,129 & 50,076 & 47,649 & 47,115 & 45,305 & 47,687 \\
\hline 2018 & 552,029 & 268,779 & 283,250 & 47,759 & 42,564 & 42,564 & 45,920 & 42,400 & 45,098 & 45,038 & 46,945 & 49,705 & 46,738 & 47,015 & 45,707 & 47,140 \\
\hline $\begin{array}{l}\text { American } \\
\text { Indian or } \\
\text { Alaska Native: }\end{array}$ & & & & & & & & & & & & & & & & \\
\hline $2020^{3}$ & 26,638 & 13,218 & 13,420 & 2,292 & 2,049 & 1,978 & 2,209 & 2,192 & 2,235 & 2,241 & 2,410 & 2,414 & 2,315 & 2,211 & 1,982 & 2,088 \\
\hline 2019 & 28,450 & 14,013 & 14,437 & 2,525 & 2,182 & 2,182 & 2,332 & 2,293 & 2,382 & 2,299 & 2,518 & 2,521 & 2,462 & 2,377 & 2,170 & 2,389 \\
\hline 2018 & 29,092 & 14,038 & 15,054 & 2,427 & 2,230 & 2,230 & 2,454 & 2,140 & 2,361 & 2,426 & 2,589 & 2,740 & 2,432 & 2,504 & 2,378 & 2,411 \\
\hline \multicolumn{17}{|l|}{ Asian: } \\
\hline $2020^{3}$ & 218,860 & 110,791 & 108,069 & 19,303 & 17,676 & 17,066 & 19,265 & 17,980 & 18,691 & 17,876 & 18,984 & 18,771 & 18,122 & 18,554 & 17,273 & 16,365 \\
\hline 2019 & 238,769 & 116,289 & 122,480 & 19,628 & 17,975 & 17,975 & 19,910 & 19,261 & 20,168 & 19,347 & 20,473 & 21,021 & 19,777 & 21,159 & 19,832 & 20,218 \\
\hline 2018 & 240,798 & 118,836 & 121,962 & 20,639 & 18,143 & 18,143 & 20,413 & 19,354 & 20,641 & 19,646 & 20,252 & 21,049 & 19,951 & 20,592 & 20,104 & 20,014 \\
\hline \multicolumn{17}{|l|}{$\begin{array}{l}\text { Native } \\
\text { Hawaiian } \\
\text { or Pacific } \\
\text { Islander: }\end{array}$} \\
\hline $2020^{3}$ & 9,612 & 4,660 & 4,952 & 802 & 786 & 759 & 792 & 704 & 819 & 757 & 836 & 850 & 800 & 824 & 818 & 824 \\
\hline 2019 & 9,770 & 4,695 & 5,075 & 790 & 762 & 762 & 814 & 738 & 847 & 744 & 843 & 888 & 815 & 852 & 792 & 885 \\
\hline 2018 & 9,476 & 4,647 & 4,829 & 790 & 686 & 686 & 810 & 786 & 796 & 779 & 803 & 839 & 805 & 794 & 801 & 787 \\
\hline \multicolumn{17}{|l|}{ Hispanic $^{5}$} \\
\hline $2020^{3}$ & 863,949 & 420,419 & 443,530 & 73,597 & 67,461 & 65,135 & 70,334 & 67,958 & 70,051 & 71,018 & 76,930 & 77,366 & 76,710 & 74,543 & 69,581 & 68,400 \\
\hline 2019 & 886,467 & 421,991 & 464,476 & 73,742 & 65,667 & 65,667 & 70,442 & 68,517 & 72,747 & 70,876 & 78,805 & 81,983 & 79,240 & 78,074 & 71,532 & 74,842 \\
\hline 2018 & 886,210 & 424,400 & 461,810 & 74,247 & 66,039 & 66,039 & 72,088 & 67,276 & 72,745 & 72,005 & 77,446 & 81,472 & 77,294 & 77,416 & 73,576 & 74,606 \\
\hline
\end{tabular}




\section{Vital Statistics Surveillance Report}

Table 1. Births, by race and Hispanic origin of the mother and month of birth: United States, final 2018 and 2019 and provisional 2020, and percent change in births from 2018-2019 and 2019-2020-Con.

\begin{tabular}{|c|c|c|c|c|c|c|c|c|c|c|c|c|c|c|c|c|}
\hline $\begin{array}{c}\text { Race and } \\
\text { Hispanic origin } \\
\text { of mother and } \\
\text { year }\end{array}$ & Total & $\begin{array}{l}\text { January- } \\
\text { June }\end{array}$ & $\begin{array}{c}\text { July- } \\
\text { December }\end{array}$ & January & $\begin{array}{l}\text { February } \\
\text { (observed) }\end{array}$ & $\begin{array}{l}\text { February } \\
\text { (adjusted) }^{1}\end{array}$ & March & April & May & June & July & August & September & October & November & December \\
\hline $\begin{array}{c}\text { All races and } \\
\text { origins }^{2}\end{array}$ & \multicolumn{16}{|c|}{ Percent change } \\
\hline 2019-2020 & -4 & -2 & -6 & -2 & 1 & -3 & -1 & -3 & -5 & -1 & -4 & -7 & -4 & -6 & -6 & -8 \\
\hline 2018-2019 & -1 & -2 & -1 & -1 & -2 & -2 & -4 & †0 & -1 & -3 & 1 & -1 & 1 & -1 & -3 & -1 \\
\hline \multicolumn{17}{|l|}{$\begin{array}{l}\text { Non-Hispanic, } \\
\text { single race }\end{array}$} \\
\hline \multicolumn{17}{|l|}{ White: } \\
\hline 2019-2020 & -4 & -2 & -6 & -3 & to & -3 & -1 & -4 & -5 & -1 & -4 & -7 & -5 & -7 & -6 & -6 \\
\hline 2018-2019 & -2 & -2 & -2 & -2 & -2 & -2 & -4 & $\dagger-1$ & -2 & -4 & 1 & -2 & †0 & -2 & -5 & -2 \\
\hline \multicolumn{17}{|l|}{ Black: } \\
\hline 2019-2020 & -4 & -1 & -6 & -2 & $\dagger 1$ & -2 & †0 & -2 & -4 & $\dagger 1$ & -4 & -7 & -5 & -6 & -6 & -8 \\
\hline 2018-2019 & -1 & -2 & 1 & $t-1$ & -3 & -3 & -5 & $\dagger-1$ & $t-1$ & -5 & 3 & $\dagger 1$ & 2 & †0 & $\dagger-1$ & $\dagger 1$ \\
\hline \multicolumn{17}{|l|}{$\begin{array}{l}\text { American } \\
\text { Indian or } \\
\text { Alaska Native: }\end{array}$} \\
\hline 2019-2020 & -6 & -6 & -7 & -9 & -6 & -9 & $\dagger-5$ & $\dagger-4$ & -6 & †-3 & $\dagger-4$ & $\dagger-4$ & -6 & -7 & -9 & -13 \\
\hline 2018-2019 & -2 & to & -4 & $\dagger 4$ & $\dagger-2$ & $\dagger-2$ & $\dagger-5$ & 7 & $\dagger 1$ & $\dagger-5$ & $t-3$ & -8 & $\dagger 1$ & $\dagger-5$ & -9 & t-1 \\
\hline \multicolumn{17}{|l|}{ Asian: } \\
\hline 2019-2020 & -8 & -5 & -12 & $t-2$ & $\dagger-2$ & -5 & -3 & -7 & -7 & -8 & -7 & -11 & -8 & -12 & -13 & -19 \\
\hline 2018-2019 & -1 & -2 & †0 & -5 & $\dagger-1$ & $\dagger-1$ & -2 & †0 & -2 & $\dagger-2$ & $\dagger 1$ & †0 & $\dagger-1$ & 3 & $\dagger-1$ & $\dagger 1$ \\
\hline \multicolumn{17}{|l|}{$\begin{array}{l}\text { Native } \\
\text { Hawaiian } \\
\text { or Pacific } \\
\text { Islander: }\end{array}$} \\
\hline 2019-2020 & $\dagger-2$ & $\dagger-1$ & $\dagger-2$ & $\dagger 2$ & $\dagger 3$ & †0 & $\dagger-3$ & $\dagger-5$ & t-3 & $\dagger 2$ & $\dagger-1$ & $\dagger-4$ & $\dagger-2$ & t-3 & $\dagger 3$ & $\dagger-7$ \\
\hline 2018-2019 & 3 & $\dagger 1$ & 5 & to & 11 & 11 & to & $\dagger-6$ & +6 & $\dagger-4$ & +5 & +6 & $\dagger 1$ & $\dagger 7$ & $t-1$ & 12 \\
\hline \multicolumn{17}{|l|}{ Hispanic ${ }^{5}$} \\
\hline 2019-2020 & -3 & †0 & -5 & †0 & 3 & $t-1$ & †0 & $\dagger-1$ & -4 & †0 & -2 & -6 & -3 & -5 & -3 & -9 \\
\hline 2018-2019 & †0 & -1 & 1 & $\dagger-1$ & $\dagger-1$ & $t-1$ & -2 & 2 & to & -2 & 2 & $\dagger 1$ & 3 & $\dagger 1$ & -3 & †0 \\
\hline
\end{tabular}

$\dagger$ No significant change $(p<0.05)$.
0 Quantity equal to or more than zero but less than 0.5 .

${ }^{1}$ Number of births for February 2020 was adjusted for leap day, February 29.

"Includes births to race and origin groups not shown separately, such as Hispanic, single-race white; Hispanic, single-race black; and non-Hispanic, multiple-race women; and births with origin not stated.

${ }^{3}$ Provisional 2020 data; based on $99.87 \%$ of births.

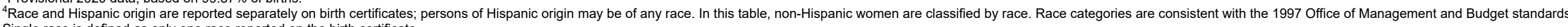
Single race is defined as only one race reported on the birth certificate.

SOURCE: National Center for Health Statistics, National Vital Statistics System, Natality. 


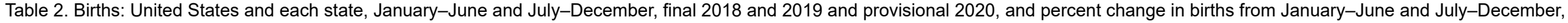
2018-2019 and 2019-2020

\begin{tabular}{|c|c|c|c|c|c|c|c|c|c|}
\hline \multirow[b]{2}{*}{ Reporting area } & \multicolumn{3}{|c|}{2018} & \multicolumn{3}{|c|}{2019} & \multicolumn{3}{|c|}{$2020^{1}$} \\
\hline & Total & January-June & July-December & Total & January-June & July-December & Total & January-June & July-December \\
\hline & \multicolumn{9}{|c|}{ Number } \\
\hline Total & $3,791,712$ & $1,848,934$ & $1,942,778$ & $3,747,540$ & $1,814,497$ & $1,933,043$ & $3,605,201$ & $1,782,237$ & $1,822,964$ \\
\hline Alabama & 57,761 & 27,951 & 29,810 & 58,615 & 27,778 & 30,837 & 57,634 & 28,052 & 29,582 \\
\hline Alaska & 10,086 & 5,073 & 5,013 & 9,822 & 4,881 & 4,941 & 9,447 & 4,706 & 4,741 \\
\hline Arizona & 80,723 & 38,641 & 42,082 & 79,375 & 38,168 & 41,207 & 76,923 & 37,439 & 39,484 \\
\hline Arkansas & 37,018 & 17,965 & 19,053 & 36,564 & 17,439 & 19,125 & 35,210 & 17,185 & 18,025 \\
\hline California & 454,920 & 220,137 & 234,783 & 446,479 & 216,456 & 230,023 & 419,612 & 207,015 & 212,597 \\
\hline Colorado & 62,885 & 30,872 & 32,013 & 62,869 & 30,590 & 32,279 & 61,493 & 30,528 & 30,965 \\
\hline Connecticut & 34,725 & 17,022 & 17,703 & 34,258 & 16,752 & 17,506 & 33,448 & 16,613 & 16,835 \\
\hline Delaware & 10,621 & 5,253 & 5,368 & 10,562 & 4,963 & 5,599 & 10,336 & 5,050 & 5,286 \\
\hline District of Columbia & 9,212 & 4,500 & 4,712 & 9,079 & 4,364 & 4,715 & 8,858 & 4,461 & 4,397 \\
\hline Florida & 221,542 & 106,482 & 115,060 & 220,002 & 105,184 & 114,818 & 209,612 & 102,477 & 107,135 \\
\hline Georgia & 126,172 & 61,278 & 64,894 & 126,371 & 60,855 & 65,516 & 122,266 & 60,027 & 62,239 \\
\hline Hawaii & 16,972 & 8,376 & 8,596 & 16,797 & 8,191 & 8,606 & 15,730 & 7,827 & 7,903 \\
\hline Idaho & 21,403 & 10,547 & 10,856 & 22,063 & 10,896 & 11,167 & 21,520 & 10,642 & 10,878 \\
\hline lowa & 37,785 & 18,679 & 19,106 & 37,649 & 18,518 & 19,131 & 36,080 & 18,018 & 18,062 \\
\hline Kansas & 36,261 & 17,716 & 18,545 & 35,395 & 17,130 & 18,265 & 34,360 & 16,922 & 17,438 \\
\hline Kentucky & 53,922 & 26,160 & 27,762 & 53,069 & 25,520 & 27,549 & 51,581 & 25,215 & 26,366 \\
\hline Louisiana & 59,615 & 28,686 & 30,929 & 58,941 & 27,849 & 31,092 & 57,070 & 27,631 & 29,439 \\
\hline Maine & 12,311 & 5,962 & 6,349 & 11,779 & 5,772 & 6,007 & 11,532 & 5,683 & 5,849 \\
\hline Maryland & 71,080 & 34,508 & 36,572 & 70,178 & 34,035 & 36,143 & 68,523 & 34,010 & 34,513 \\
\hline Massachusetts & 69,109 & 34,176 & 34,933 & 69,117 & 33,601 & 35,516 & 66,429 & 33,376 & 33,053 \\
\hline Michigan & 110,032 & 54,444 & 55,588 & 107,886 & 53,014 & 54,872 & 103,846 & 52,249 & 51,597 \\
\hline Minnesota & 67,344 & 33,357 & 33,987 & 66,027 & 32,474 & 33,553 & 63,387 & 31,797 & 31,590 \\
\hline Mississippi & 37,000 & 17,828 & 19,172 & 36,636 & 17,233 & 19,403 & 35,457 & 17,190 & 18,267 \\
\hline Missouri & 73,269 & 35,912 & 37,357 & 72,127 & 35,046 & 37,081 & 69,238 & 34,232 & 35,006 \\
\hline Montana & 11,513 & 5,811 & 5,702 & 11,079 & 5,546 & 5,533 & 10,785 & 5,498 & 5,287 \\
\hline Nebraska & 25,488 & 12,489 & 12,999 & 24,755 & 12,152 & 12,603 & 24,235 & 12,080 & 12,155 \\
\hline Nevada & 35,682 & 17,219 & 18,463 & 35,072 & 16,798 & 18,274 & 33,632 & 16,350 & 17,282 \\
\hline New Hampshire & 11,995 & 5,865 & 6,130 & 11,839 & 5,732 & 6,107 & 11,773 & 5,785 & 5,988 \\
\hline New Jersey & 101,223 & 49,612 & 51,611 & 99,585 & 48,245 & 51,340 & 96,543 & 48,387 & 48,156 \\
\hline New Mexico & 23,039 & 11,033 & 12,006 & 22,960 & 11,048 & 11,912 & 21,316 & 10,739 & 10,577 \\
\hline New York & 226,238 & 111,416 & 114,822 & 221,539 & 107,791 & 113,748 & 209,172 & 105,506 & 103,666 \\
\hline Ohio & 135,134 & 66,471 & 68,663 & 134,461 & 65,303 & 69,158 & 129,071 & 63,862 & 65,209 \\
\hline
\end{tabular}


Vital Statistics Surveillance Report

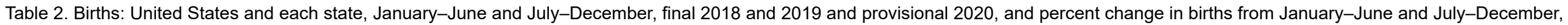
2018-2019 and 2019-2020-Con.

\begin{tabular}{|c|c|c|c|c|c|c|c|c|c|}
\hline \multirow[b]{2}{*}{ Reporting area } & \multicolumn{3}{|c|}{2018} & \multicolumn{3}{|c|}{2019} & \multicolumn{3}{|c|}{$2020^{1}$} \\
\hline & Total & January-June & July-December & Total & January-June & July-December & Total & January-June & July-December \\
\hline & \multicolumn{9}{|c|}{ Number } \\
\hline Oklahoma & 49,800 & 24,237 & 25,563 & 49,143 & 23,607 & 25,536 & 47,393 & 23,286 & 24,107 \\
\hline Oregon & 42,188 & 20,907 & 21,281 & 41,858 & 20,937 & 20,921 & 39,792 & 19,993 & 19,799 \\
\hline Pennsylvania & 135,673 & 66,571 & 69,102 & 134,230 & 65,204 & 69,026 & 130,562 & 64,658 & 65,904 \\
\hline Rhode Island & 10,506 & 5,184 & 5,322 & 10,175 & 4,993 & 5,182 & 10,102 & 5,066 & 5,036 \\
\hline South Dakota & 11,893 & 5,999 & 5,894 & 11,449 & 5,686 & 5,763 & 10,952 & 5,519 & 5,433 \\
\hline Tennessee & 80,751 & 39,280 & 41,471 & 80,450 & 38,715 & 41,735 & 78,659 & 38,240 & 40,419 \\
\hline Texas & 378,624 & 182,268 & 196,356 & 377,599 & 179,973 & 197,626 & 365,857 & 178,369 & 187,488 \\
\hline Utah & 47,209 & 23,275 & 23,934 & 46,826 & 23,175 & 23,651 & 45,702 & 23,084 & 22,618 \\
\hline Vermont & 5,432 & 2,622 & 2,810 & 5,361 & 2,689 & 2,672 & 5,117 & 2,574 & 2,543 \\
\hline Virginia & 99,843 & 48,938 & 50,905 & 97,429 & 47,295 & 50,134 & 94,391 & 46,848 & 47,543 \\
\hline Washington & 86,085 & 42,529 & 43,556 & 84,895 & 42,165 & 42,730 & 83,067 & 41,481 & 41,586 \\
\hline West Virginia & 18,248 & 9,046 & 9,202 & 18,136 & 8,767 & 9,369 & 17,159 & 8,510 & 8,649 \\
\hline Wisconsin & 64,098 & 31,604 & 32,494 & 63,270 & 31,144 & 32,126 & 60,491 & 30,528 & 29,963 \\
\hline
\end{tabular}




\section{Vital Statistics Surveillance Report}

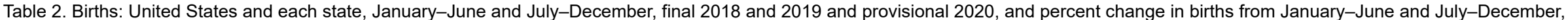
2018-2019 and 2019-2020-Con.

\begin{tabular}{|c|c|c|c|c|c|}
\hline \multirow[b]{2}{*}{ Reporting area } & \multicolumn{3}{|c|}{ 2018-2019 } & \multicolumn{2}{|c|}{ 2019-2020 } \\
\hline & Total & January-June & July-December & Total & July-December \\
\hline
\end{tabular}

Total

Alabama

Alaska

Arizona

Arkansas

California

Colorado

Connecticut

Delaware

District of Columbia

Florida

Georgia

Hawaii

Idaho

Illinois

Indiana

lowa

Kansas

Kentucky

Louisiana

Maine

Maryland

Massachusetts

Michigan

Minnesota

Mississippi

Missouri

Montana

Nebraska

Nevada

Total

January-June

Percent change

Percent change

$\begin{array}{rr}-2 & -1 \\ \dagger-1 & 3 \\ \dagger-4 & \dagger-1 \\ \dagger-1 & -2 \\ -3 & +0 \\ -2 & -2 \\ \dagger-1 & \dagger 1 \\ \dagger-2 & \dagger-1 \\ -6 & 4 \\ \dagger-3 & \dagger 0 \\ -1 & +0 \\ \dagger-1 & \dagger 1 \\ \dagger-2 & \dagger 0 \\ 3 & 3 \\ -5 & -2 \\ -3 & \dagger 1 \\ \dagger-1 & \dagger 0 \\ -3 & \dagger-2 \\ -2 & \dagger-1 \\ -3 & \dagger 1 \\ \dagger-3 & -5 \\ \dagger-1 & \dagger-1 \\ -2 & 2 \\ -3 & -1 \\ -3 & \dagger-1 \\ -3 & \dagger 1 \\ -2 & \dagger-1 \\ -5 & \dagger-3 \\ -3 & -3 \\ -2 & \dagger-1\end{array}$

$\begin{array}{rr}-1 & -2 \\ 1 & \dagger-1 \\ \dagger-3 & \dagger-4 \\ -2 & \dagger-1 \\ \dagger-1 & -3 \\ -2 & -2 \\ \dagger 0 & \dagger-1 \\ \dagger-1 & \dagger-2 \\ \dagger-1 & -6 \\ \dagger-1 & \dagger-3 \\ -1 & -1 \\ \dagger 0 & \dagger-1 \\ \dagger-1 & \dagger-2 \\ 3 & 3 \\ -3 & -5 \\ \dagger-1 & -3 \\ \dagger 0 & \dagger-1 \\ -2 & -3 \\ -2 & -2 \\ \dagger-1 & -3 \\ -4 & \dagger-3 \\ -1 & \dagger-1 \\ \dagger 0 & -2 \\ -2 & -3 \\ -2 & -3 \\ \dagger-1 & -3 \\ -2 & -2 \\ -4 & -5 \\ -3 & -3 \\ -2 & -2 \\ & \end{array}$

$\begin{array}{rr}-1 & -4 \\ 3 & -2 \\ -1 & -4 \\ -2 & -3 \\ \dagger 0 & -4 \\ -2 & -6 \\ \dagger 1 & -2 \\ \dagger-1 & -2 \\ 4 & \dagger-2 \\ \dagger 0 & \dagger-2 \\ \dagger 0 & -5 \\ \dagger 1 & -3 \\ \dagger 0 & -6 \\ 3 & -2 \\ -2 & -5 \\ \dagger 1 & -3 \\ \dagger 0 & -4 \\ \dagger-2 & -3 \\ \dagger-1 & -3 \\ \dagger 1 & -3 \\ -5 & \dagger-2 \\ \dagger-1 & -2 \\ 2 & -4 \\ -1 & -4 \\ \dagger-1 & -4 \\ \dagger 1 & -3 \\ \dagger-1 & -4 \\ -3 & -3 \\ -3 & -2 \\ -1 & -4\end{array}$

$\begin{array}{rr}-4 & -2 \\ -2 & \dagger 1 \\ -4 & \dagger-4 \\ -3 & -2 \\ -4 & \dagger-1 \\ -6 & -4 \\ -2 & \dagger 0 \\ -2 & \dagger-1 \\ \dagger-2 & \dagger 2 \\ \dagger-2 & \dagger 2 \\ -5 & -3 \\ -3 & -1 \\ -6 & -4 \\ -2 & \dagger-2 \\ -5 & -3 \\ -3 & \dagger 0 \\ -4 & -3 \\ -3 & \dagger-1 \\ -3 & \dagger-1 \\ -3 & \dagger-1 \\ \dagger-2 & \dagger-2 \\ -2 & \dagger 0 \\ -4 & \dagger-1 \\ -4 & -1 \\ -4 & -2 \\ -3 & \dagger 0 \\ -4 & -2 \\ -3 & \dagger-1 \\ -2 & \dagger-1 \\ -4 & -3\end{array}$




\section{Vital Statistics Surveillance Report}

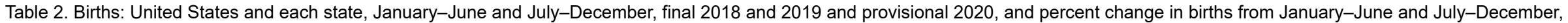
2018-2019 and 2019-2020-Con.

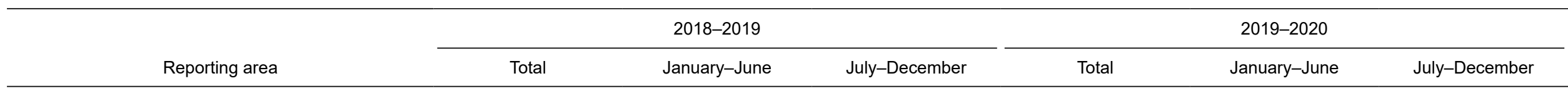

New Hampshire

New Jersey

New Mexico

New York

North Carolina

North Dakota

Ohio

Oklahoma

Oregon

Pennsylvania

Rhode Island

South Carolina

South Dakota

Tennessee

Texas

Utah

Vermont

Virginia

Washington

West Virginia

Wisconsin

Wyoming

$t-1$
-2
+0
-2
+0
$t-2$
+0
-1
$t-1$
-1
-3
+1
-4
+0
+0
$t-1$
+-1
-2
-1
+-1
-1
+0

Percent change

$\dagger$ No significant change $(p<0.05)$

0 Quantity more than zero but less than 0.5 .

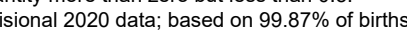

SOURCE: National Center for Health Statistics, National Vital Statistics System, Natality. 


\section{Acknowledgments}

This report was prepared in the Division of Vital Statistics (DVS) under the general direction of Steven Schwartz, Director; Isabelle Horon, Chief, Reproductive Statistics Branch (RSB); and Joyce Martin, Team Leader, RSB Birth Team. Rajesh Virkar, Chief, Information Technology Branch (ITB), and Jasmine N. Mickens, Steve J.

Steimel, Annie S. Liu, and Legesse Alemu provided computer programming support and statistical tables. Jasmine N. Mickens, Steve J. Steimel, and Annie $\mathrm{S}$. Liu of ITB prepared the natality file. The Registration Methods and Data Acquisition, Classification and Evaluation Branch staff of DVS evaluated the quality of and acceptance procedures for the state data files on which this report is based. Danielle M. Ely of RSB provided content review.

\section{Suggested citation}

Hamilton BE, Osterman MJK, Martin JA. Declines in births by month: United States, 2020. Vital Statistics Rapid Release; no 14. Hyattsville, MD: National Center for Health Statistics. June 2021. DOI: https://dx.doi.org/10.15620/ cdc:106116.

\section{Copyright information}

All material appearing in this report is in the public domain and may be reproduced or copied without permission; citation as to source, however, is appreciated.

\section{National Center for Health Statistics}

Brian C. Moyer, Ph.D., Director

Amy M. Branum, Ph.D., Acting Associate Director for Science

\section{Division of Vital Statistics}

Steven Schwartz, Ph.D., Director

Isabelle Horon, Dr.P.H., Acting Associate Director for Science 